\title{
Papers presented at the Association of Otolaryngologists in Training Annual Meeting, 20 June 2014, Exeter, UK
}

\author{
President: Mr Zaid Awad, North Thames Deanery (ENT) \\ Secretary: Mr Richard Williams, South West Peninsula Deanery (ENT) \\ Treasurer: Ms Natalie Ronan, South West Peninsula Deanery (ENT) \\ Invited panel: Mr S A Hickey, Torbay Hospital; Prof H Khalil, Derriford Hospital; Mr A Evans, Great Yarmouth and Waveney \\ Clinical Commissioning Group; Prof T Jones, Aintree University Hospital; Mr D Skinner, Shrewsbury and Telford Hospital; Prof \\ B N Kumar, Royal Albert Edward Infirmary (Wigan); Mr J Rainsbury, Derriford Hospital; Prof A Narula, St Mary’s Hospital \\ (London); Mr J Powles, Torbay Hospital \\ Junior Otolaryngologist in Training prize (presented by Prof Narula) was awarded to Winnie Yeung and Helena Wilson for 'Quality improve- \\ ment without significant costs: improving rates of day-case tonsillectomies in the absence of a dedicated day-case unit'.
}

Investigating the parotid tumour: are magnetic resonance imaging scans more accurate in diagnosing malignant parotid tumours than fine needle aspiration cytology?

M Bajalan ${ }^{1}$, T Biggs $^{2}, \mathrm{~S}$ Jayaram $^{1}$, P Pracy $^{1}$

From the ${ }^{1}$ University Hospital Birmingham NHS Foundation Trust and the ${ }^{2}$ University Hospital Southampton NHS Foundation Trust

\section{Background}

The key step in the evaluation of parotid lumps is to differentiate benign lesions from malignant ones. The ENT-UK guidelines advocate the use of fine needle aspiration cytology (FNAC). There are minimal evidence-based data concerning alternative diagnostic tools.

\section{Objective}

We decided to investigate the accuracy of magnetic resonance imaging (MRI) compared with FNAC in correctly diagnosing malignant parotid tumours.

\section{Methods}

All patients who had undergone parotidectomy from January 2012 to June 2013 were identified. The parotid sample list was acquired from the pathology department. Only parotidectomies carried out for primary tumour removal were considered; all parotidectomies performed as part of a complete dissection for other primary malignancies were excluded.

Retrospective analysis identified the investigations that took place prior to surgery. The results of each test were compared to the definitive histopathology result, which was considered the 'gold standard' diagnosis.
Results

\begin{tabular}{|c|c|c|c|}
\hline PREDICTIVE A & $\begin{array}{r}\text { TABL } \\
\text { TY OF MR } \\
\text { MASS DIAC }\end{array}$ & $\begin{array}{l}\text { I } \\
\text { AND FI } \\
\text { NOSIS }\end{array}$ & C IN PAROTID \\
\hline $\begin{array}{l}\text { MRI \& FNAC } \\
\text { diagnosis }\end{array}$ & $\begin{array}{r}\text { Tissue pa } \\
\text { diagn }\end{array}$ & $\begin{array}{l}\text { hology } \\
\text { sis }\end{array}$ & $\begin{array}{c}\text { Predictive value } \\
(\%)\end{array}$ \\
\hline & Malignant & Benign & \\
\hline MRI & & & \\
\hline - Malignant & 6 & 1 & $\mathrm{PPV}=85.7$ \\
\hline - Benign & 1 & 19 & $\mathrm{NPV}=95$ \\
\hline - Sensitivity (\%) & 85.7 & - & \\
\hline - Specificity (\%) & - & 95 & \\
\hline $\begin{array}{l}\text { FNAC } \\
\text { - Malignant }\end{array}$ & 4 & 0 & $\mathrm{PPV}=100$ \\
\hline - Benign & 3 & 19 & $\mathrm{NPV}=86$ \\
\hline - Sensitivity (\%) & 57.1 & - & \\
\hline - Specificity (\%) & - & 100 & \\
\hline
\end{tabular}

Data represent numbers of patients unless indicated otherwise. MRI = magnetic resonance imaging; FNAC $=$ fine needle aspiration cytology; PPV = positive predictive value; $\mathrm{NPV}=$ negative predictive value

\section{Conclusion}

Accurate pre-operative assessment of benign versus malignant parotid lumps helps the surgeon to plan excision and determine the need for neck dissection; it can also significantly alter the incidence of peri-operative facial nerve paresis or paralysis. 
Our results highlight the sensitivity of MRI as a diagnostic tool in differentiating malignant from benign parotid lesions. Fine needle aspiration cytology demonstrates significant accuracy in benign lesions, but appears limited for malignant pathology. There may be a substantial role for MRI; we feel this should be explored by analysing a larger cohort of malignant parotid tumour data, the outcomes of which may have a potentially significant impact on changing current recommended practice.

Quality improvement without significant costs:

improving rates of day-case tonsillectomies in the absence of a dedicated day-case unit

W Yeung, H Wilson

From the Royal Devon and Exeter Hospital

\section{Background and objective}

Over 55000 tonsillectomies are performed in England every year. Historically, patients stay overnight. The Royal College of Surgeons deemed tonsillectomies inappropriate for daycase surgery in 1985, citing reactionary haemorrhage as the contraindication. Since then, published evidence has concluded that this risk is low, prompting a shift in practice. We present the challenges of improving rates of day-case tonsillectomies in the absence of a dedicated day-case unit.

\section{Methods}

Data on day-case tonsillectomy rates in both children and adults were collected over six months in $2011(n=123)$. New local protocols were designed specifically for daycase tonsillectomies. Day-case tonsillectomy rates were reaudited over six months in $2012(n=125)$ to assess the effect of intervention.

\section{Results}

Day-case tonsillectomy rates increased in children from 9 to 25 per cent. A similarly significant increase was noted in adults, from 6 to 43 per cent. There were two re-admissions over the re-audit period, neither of which involved day-case patients.

\section{Conclusion}

This project demonstrates how healthcare processes can be streamlined to save time and resources without compromising patient safety. By engaging colleagues and patients, we made dramatic improvements, without needing to construct an expensive, purpose-built day-case facility, which is all the more relevant in times of efficiency savings.
Procedures of limited clinical value in ENT: what effect has there been on operating numbers?

\section{F Shelton, T Biggs, A Henderson, N Patel}

From the University Hospital Southampton Foundation NHS Trust

\section{Background and objective}

'Procedures of limited clinical value' is a term first highlighted in the McKinsey report (2009), which examined potential cost-cutting measures within the future National Health Service (NHS). It is a term used by some trusts to describe surgical procedures that, they feel, are not cost effective. With the NHS now facing intense financial pressures, we undertook a study to examine whether five core procedures within the specialty of ENT had been restricted.

\section{Methods}

Numbers of operative procedures carried out over a six-year period (2007-2012) at six separate hospitals across the Wessex Deanery were obtained. Five surgical ENT procedures commonly classified as procedures of limited clinical value were studied: tonsillectomy, adenoidectomy, septorhinoplasty, pinnaplasty, and myringotomy with grommet insertion.

\section{Results}

In total, 22839 surgical procedures were included. A yearon-year variability in the number of each of the five individual procedures was observed. However, when comparing the cumulative data from pre-2009 to post-2009, there were no significant changes in the numbers of procedures carried out. On individual examination of hospitals, no significant changes were seen in the numbers of tonsillectomies, adenoidectomies and myringotomies performed. However, a significant drop in the numbers of pinnaplasty and septorhinoplasty procedures was observed in some hospitals.

\section{Conclusion}

Since the introduction of the concept of procedures of limited clinical value in 2009 , there has not been a significant drop in the number of core ENT procedures performed across the Wessex region. However, there seems to be significant inter-hospital variation with respect to some ENT procedures. With the introduction of clinical commissioning, the inter-hospital variation in surgical provision may well increase. 